\title{
Self Diagnostic Accelerometer for mission critical health monitoring of aircraft and spacecraft engines
}

\author{
John Lekki ${ }^{1}$ and Roger Tokars ${ }^{2}$ \\ NASA Glenn Research Center, Brook Park, Ohio, 44135 \\ Dave Jaros $^{3}$ and M. Terrence Riggs ${ }^{4}$ \\ Apollo Research Corp, Buffalo, NY, 14225 \\ Kenneth P. Evans ${ }^{5}$ \\ Consultant Engineer, Cincinnati, State, 45244 \\ and \\ Andrew Gyekenyesi ${ }^{6}$ \\ Ohio Aerospace Institute, Brook Park, Ohio 44135
}

\begin{abstract}
[Abstract] A self diagnostic accelerometer system has been shown to be sensitive to multiple failure modes of charge mode accelerometers. These failures include sensor structural damage, an electrical open circuit and most importantly sensor detachment. In this paper, experimental work that was performed to determine the capabilities of a self diagnostic accelerometer system while operating in the presence of various levels of mechanical noise, emulating real world conditions, is presented. The results show that the system can successfully conduct a self diagnostic routine under these conditions.
\end{abstract}

\section{Nomenclature}

SNR = Signal to Noise ratio

$A(f) \quad=$ Self diagnostic accelerometer signal as a function of frequency

$N(f) \quad=$ Accelerometer signal, composed of structural acceleration and noise as a function of frequency

$F W H M=$ Full Width Half Maximum

$f \quad=$ Frequency, $\mathrm{Hz}$

\section{Introduction}

ccelerometers are commonly utilized for health monitoring of both aircraft and spacecraft engines ${ }^{1,2}$. In aircraft Athey are used to sense vibration signatures and shock within turbine engines in order to identify problems during operation. Similarly, spacecraft utilize accelerometers to identify problems within liquid fuel propulsion system turbo pumps. By employing physics based understanding of these systems, it is possible to identify potential failures at a much earlier time thereby providing an opportunity to take corrective action. For the specific case of a vehicle health monitoring system designed to conduct corrective action based on feed-back from accelerometers, (e.g., an automated shut down of an engine), it is readily apparent that the reliability of the sensor must be ensured. This paper discusses the results of experimental tests concerning a diagnostic system developed for mission critical

${ }^{1}$ Electronics Engineer, Optical Instrumentation and NDE branch, 21000 Brookpark Rd./Mail Stop 77-1, AIAA Member.

${ }^{2}$ Electronics Engineer, Optical Instrumentation and NDE branch, 21000 Brookpark Rd./Mail Stop 77-1, AIAA Member.

${ }^{3}$ Applications Engineer, Apollo Research Corp., 2300 Walden Ave. Buffalo, NY 14225.

${ }^{4}$ Director Research \& Engineering., Apollo Research Corp., 2300 Walden Ave. Buffalo, NY 14225.

${ }^{5}$ Consultant.

${ }^{6}$ Principal Scientist and Manager, Ohio Aerospace Institute/NASA GRC, 21000 Brookpark Rd./Mail Stop 6-1. 
accelerometers. The main objective is to show the feasibility of a self diagnostic accelerometer together with the associated electronics under real-world conditions.

The Self Diagnostic Accelerometer (SDA) system actively interrogates piezoelectric accelerometers to verify the proper operation of the accelerometers. By interrogating the sensor it is possible to identify the following failure modes: a physically damaged sensor, electrical disconnection, as well as sensor detachment/loosening from the structure $^{3-9}$. The interrogation of the sensor is accomplished by driving the piezoelectric crystal with a frequency swept sinusoidal voltage and monitoring the response from the crystal so that the frequency of a particular resonance, identified in previous work ${ }^{9}$, is determined. It should be noted that the sensor's resonant frequency, which depends on the electromechanical properties of the accelerometer, is far above the specified operational frequency range. Furthermore, the resonant frequency of the sensor has been previously shown to be sensitive to the mounting torque of the sensor. Other failures such as physical damage to the sensor crystal and electrical disconnection are also both identifiable when interrogating the sensor with this type of input signal.

A drawback to using an actuation-signal to interrogate a piezoelectric accelerometer is that the interrogation signal can cause electric fatigue in the accelerometer. Over time an applied high amplitude alternating electric field can lead to electric fatigue, or degradation, of a piezoelectric crystal. ${ }^{10-14}$ One of the objectives of this research is to determine the effectiveness of a self diagnostic system that utilizes a very low voltage (i.e., 1 Volt peak-to-peak) input signal to the accelerometer in order to avoid electric fatigue. The problem with utilizing low voltage actuation signals is that with significant mechanical noise the diagnostic response can be lost in the noise. In this paper experiments are presented that were conducted using an SDA system to determine the ability of the system to perform dependably when various magnitudes of mechanical noise are introduced within the frequency band where the self diagnostic signal is located.

\section{Experimental Procedure}

\section{A. Rotor Tests}

The SDA was tested on a disk spin simulation system consisting of a stainless steal shaft having a diameter of 20 $\mathrm{mm}$ (0.7874 in.) and a length of $781 \mathrm{~mm}$ (30.75 in.), running on precision angular contact ball bearings, which are assumed to provide isotropic stiffness support. A $25.4 \mathrm{~cm}$ (10 in.) diameter Nickel alloy disk is mounted at the midspan of the shaft. The mass of the disk is $4.9 \mathrm{Kg}(10.75 \mathrm{lb})$. With this set-up the system can operate up to $6 \mathrm{k}$ rpm (note that the first critical speed is $2.6 \mathrm{k} \mathrm{rpm}$ ). Here, the rotor tests were conducted to determine the Self Diagnostic (SD) resonance at different torque levels as well as for an untorqued condition, defined as a $1 / 4$ turn loose condition. The SD accelerometer was mounted on one of the bearing supports of the rotordynamics test rig. The SDA was tested at various torque levels. Before each successive torque level, the accelerometer was loosened and then retorqued to the new level. For the first set of experiments, the test rig was not operating so no mechanical noise was generated. Three repetitions were conducted for each torque condition and data were collected using both a commercial signal analyzer and a custom designed SD microprocessor-based electronics unit. The test setup for the spectrum analyzer is shown in Figure 1, and for the SD microprocessor-based electronics in Figure 2. The torque levels included: untorqued/ 0 in-lbs, 25 in-lbs, 30 in-lbs, 35 in-lbs and 40 in-lbs.

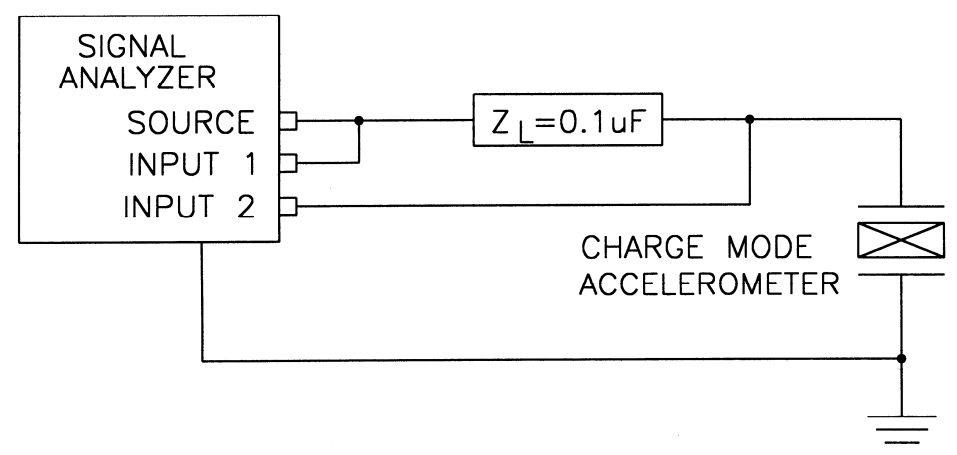

Figure 1. Test setup using the commercial signal analyzer. 
A second set of tests was conducted using the same mounting conditions but with the rotordynamics test rig operating at $3000 \mathrm{rpm}$ in order to generate mechanical noise. As was the case for the previously described tests, data was recorded using both a signal analyzer and the SD microprocessor-based electronics. These sets of data allowed for a comparison to be made between the prototype SD Electronics and the commercial signal analyzer.

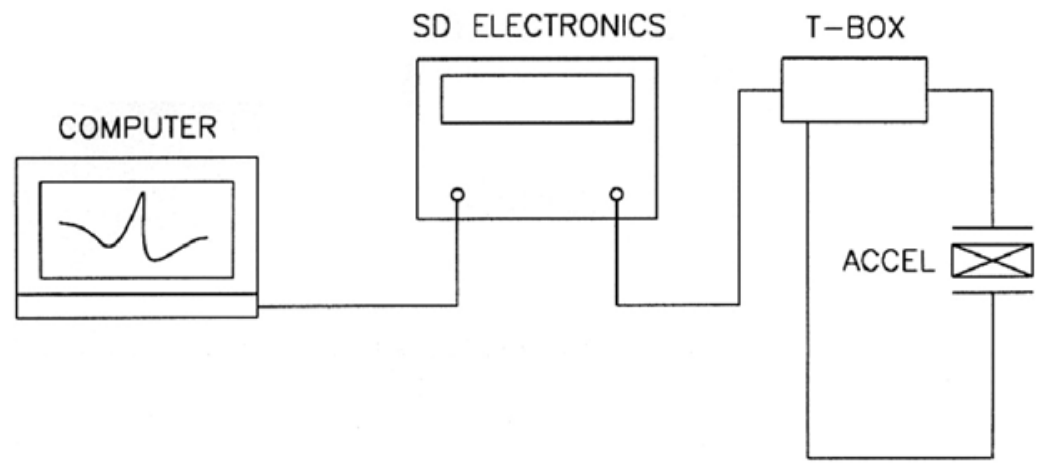

Figure 2. Test setup using the SD microprocessor-based electronics .

A flow chart summarizing the procedure is shown in Figure 3.

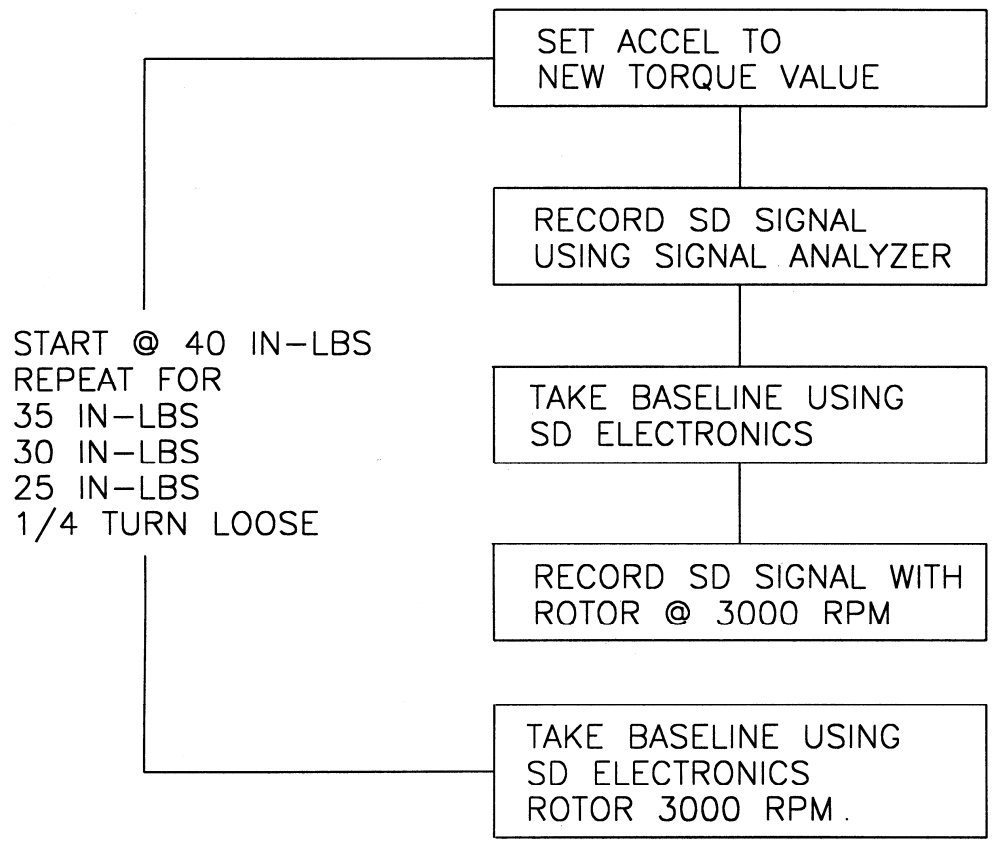

Figure 3. Flow chart for the experiments conducted using the rotor test system.

\section{B. Tests using electromechanical shaker}

The tests conducted in the rotordynamics test rig had only one level of mechanical noise, which was the noise generated by the rotor at 3000 RPM. In order to more fully characterize how the SD system works with a range of mechanical noise levels another set of experiments was conducted utilizing a shaker to generate the mechanical noise. These tests were conducted using an electromechanical shaker in accordance with the flow chart in Figure 4. The test setup is shown in Figure 5. 


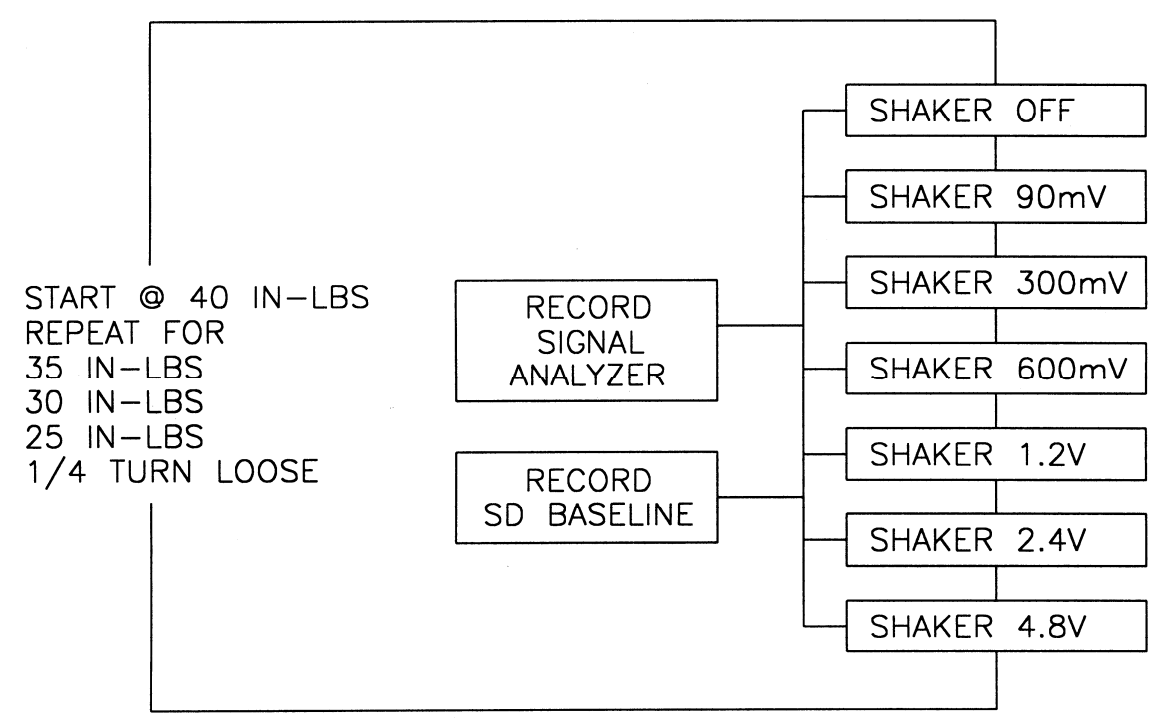

Figure 4. Flow chart for the tests performed with the electromechanical shaker.

In this test the accelerometer was mounted on a $25 \mathrm{lb}$ electromechanical shaker. The shaker vibration amplitude was controlled by a second signal analyzer coupled with a power amplifier. The control signal was chosen so as to cause mechanical vibrations at frequencies corresponding to the SD diagnostic band. The torque level of the accelerometer was also varied to the same levels as in the previous tests. This test produced a two dimensional data set of SD signals with multiple torque levels and multiple shaker amplitudes for each of the torque levels.

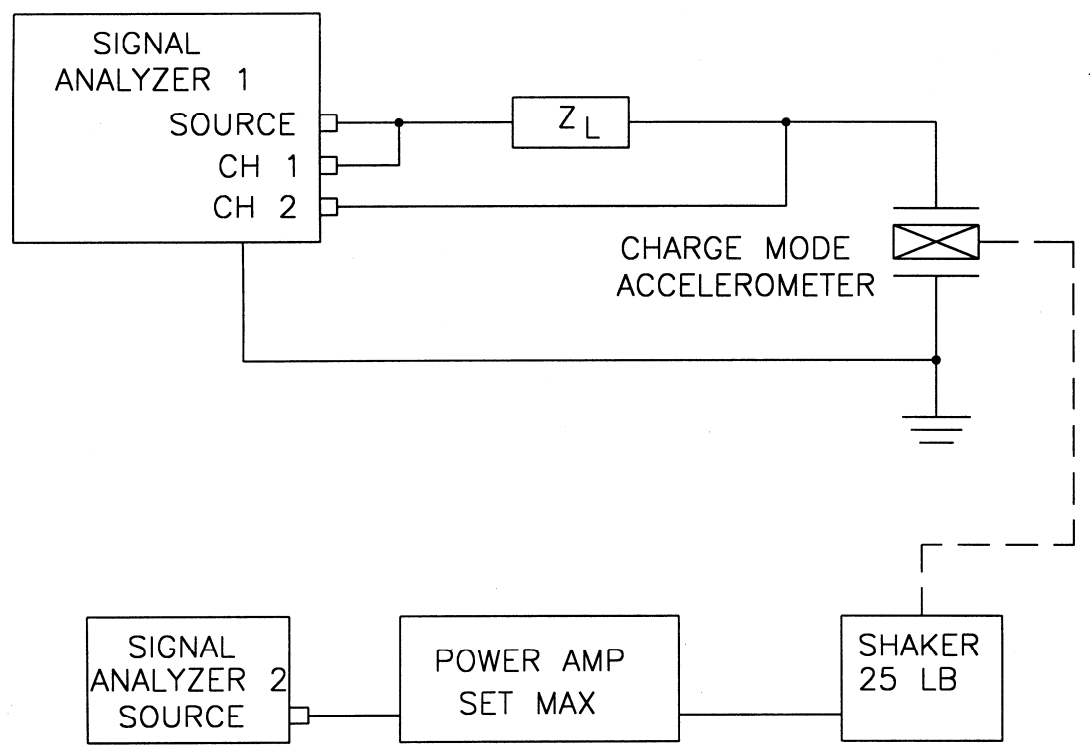

Figure 5. Test setup using the electromechanical shaker.

Next, experiments were conducted to determine the acceleration level used for the previous set of tests. The test setup is shown in Figure 6. The SD accelerometer was replaced with a reference accelerometer that had a maximum operational frequency that was higher than the diagnostic band of the accelerometer used during the SD tests. This allowed the mechanical acceleration from the shaker to be accurately measured at the diagnostic frequencies. With 
this set of data, the previous measurements of the diagnostic signal with mechanical noise could now be correlated to acceleration levels in the diagnostic frequency band, i.e., at $38 \mathrm{kHz}$.

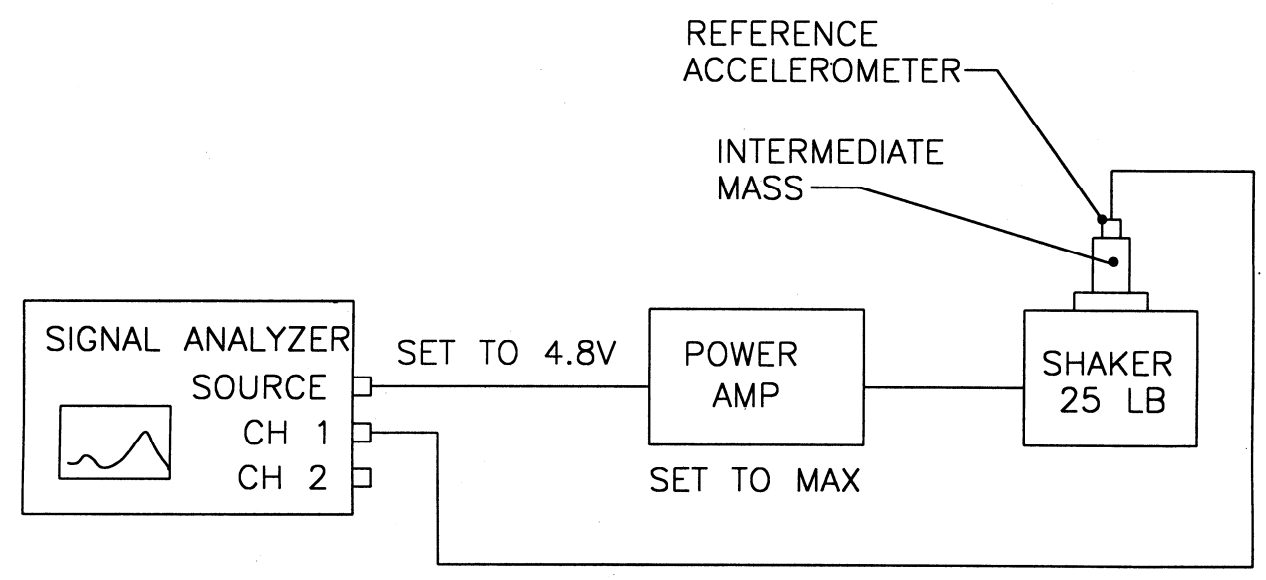

Figure 6. Test setup to determine the acceleration level used for the fourth set of tests.

\section{A. Rotor Tests}

\section{Experimental Results}

The first tests were conducted to confirm that the SD electronics, a customized electronics unit that is less expensive and complex than a commercial signal analyzer, could produce similar measurements to the signal analyzer. The SD resonant frequencies, as measured by the signal analyzer for the varied torque, are shown in Table 1. Similarly, the SD frequencies, as measured by the SD electronics, are shown in Table 2. In general, for a given torque level, the SD electronics measured a resonant frequency that was about $300 \mathrm{~Hz}$ below what was measured using the signal analyzer. These results are not problematic as long as the electronics system does not drift over time. More tests need to be conducted to observe the frequency stability of the SD electronics.

Table 1. Frequency response data using the commercial signal analyzer

\begin{tabular}{l|l|l|l} 
Torque & Resonance Freq. (Set 1) & Resonance Freq. (Set 2) & Resonance Freq. (Set 3) \\
\hline $1 / 4$ turn loose & $31.5 \mathrm{KHz}$ & $31.6 \mathrm{KHz}$ & $31.3 \mathrm{KHz}$ \\
25 in.-lbs & 34.3 & 34.3 & 34.1 \\
30 & 35.2 & 35.2 & 35.2 \\
35 & 35.5 & 35.5 & 35.4 \\
40 & 35.7 & 35.9 & 36.0
\end{tabular}

Table 2. Frequency response data using the SD microprocessor-based electronics

\begin{tabular}{l|l|l|l} 
Torque & Resonance Freq. (Set 4) & Resonance Freq. (Set 5) & Resonance Freq. (Set 6) \\
\hline 1/4 turn loose & $31.3 \mathrm{KHz}$ & $31.2 \mathrm{KHz}$ & $31.3 \mathrm{KHz}$ \\
25 in.-lbs & 34.0 & 34.0 & 34.0 \\
30 & 35.1 & 34.9 & 34.3 \\
35 & 35.1 & 35.1 & 35.2 \\
40 & 35.5 & 35.4 & 35.4
\end{tabular}

How well the SD electronics measure the frequency shift caused by varying the torque level can also be determined from this data. The average frequency shift as measured by the signal analyzer between the 40 in.-lbs 
torque and 25 in-lbs was $1.6 \mathrm{kHz}$. The measured frequency shift between the same torque levels was measured as $1.4 \mathrm{kHz}$ when using the SD electronics. Given that the variation between the maximum and minimum frequency measured for a given torque level was as much as $700 \mathrm{~Hz}$, the $200 \mathrm{~Hz}$ difference between the SD electronics and signal analyzer measured frequency shifts is not a significant difference.

With the rotor system off, baseline data was recorded using the signal analyzer. Next, data was collected with the rotor system operating at $3000 \mathrm{rpm}$. The frequency response as measured with the SD Electronics is plotted in Figure 7 as a function of frequency. This data does show that the frequency shift due to varied torque is clearly discernable in the presence of mechanical noise from the rotor operating at $3000 \mathrm{rpm}$. It was also noted, from comparison to the baseline data, that there was little or no corruption to the SD signal while operating at $3000 \mathrm{rpm}$.

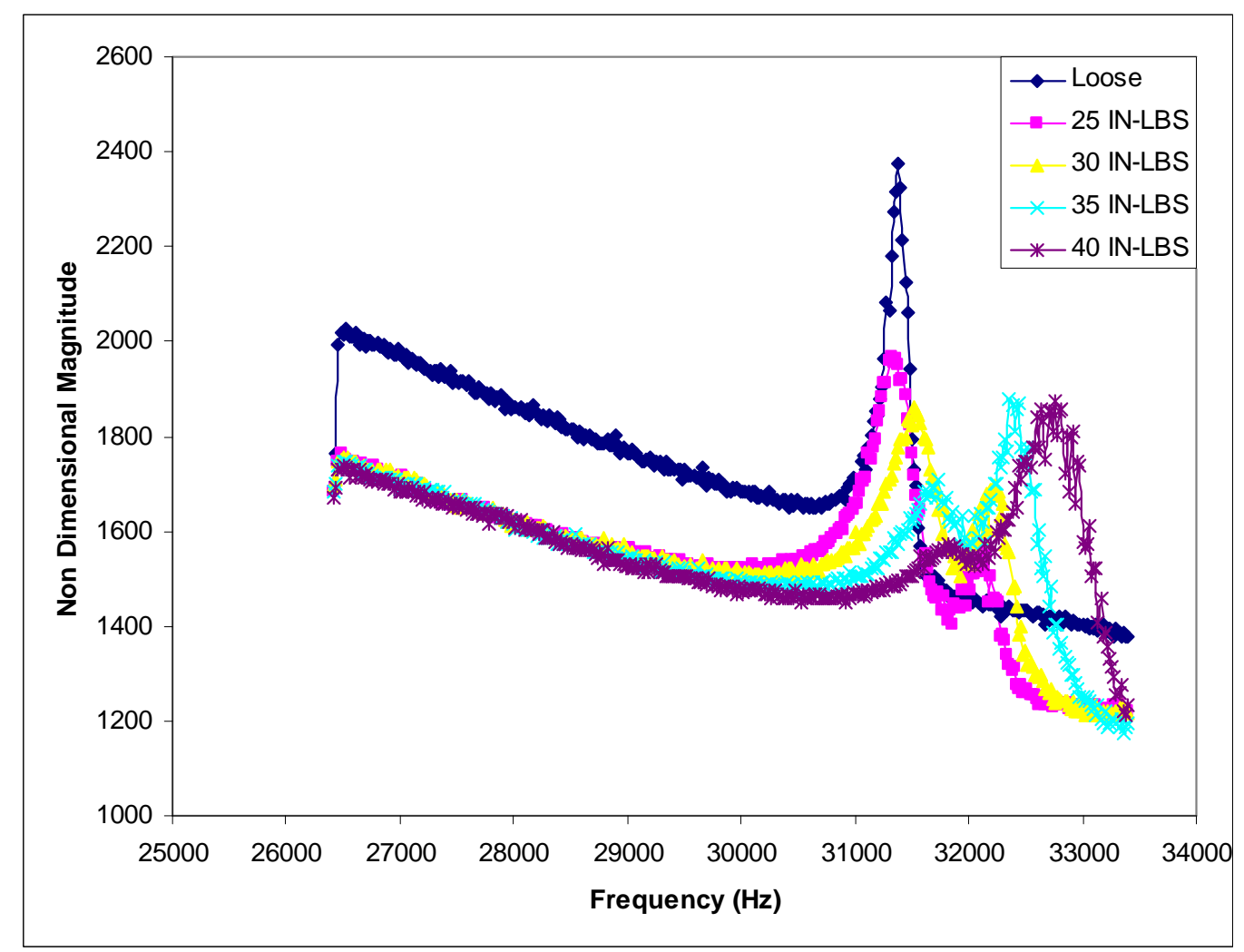

Figure 7. The SD signal at various torques with the rotor test rig operating at $3000 \mathrm{rpm}$.

\section{B. Tests using electromechanical shaker}

The next set of tests was conducted using the shaker system. Input to the shaker was configured for random noise at voltage levels of $90 \mathrm{mV}, 300 \mathrm{mV}, 600 \mathrm{mV}, 1.2 \mathrm{~V}, 2.4 \mathrm{~V}$ and $4.8 \mathrm{~V}$ over the frequency range of $30 \mathrm{KHz}$ to $40 \mathrm{KHz}$. Tests for the loose condition were only performed under two conditions; with the shaker operating at zero volts and at the maximum 4.8 volt level. Corruption of the SD signal became apparent at the $90 \mathrm{mV}$ level. This corruption of the SD signal increased with increasing vibration levels. A sample of the data illustrating this is shown in Figure 8. However, the corruption did not affect the SD signal to the point where it gave false indications when using the SD electronics. 

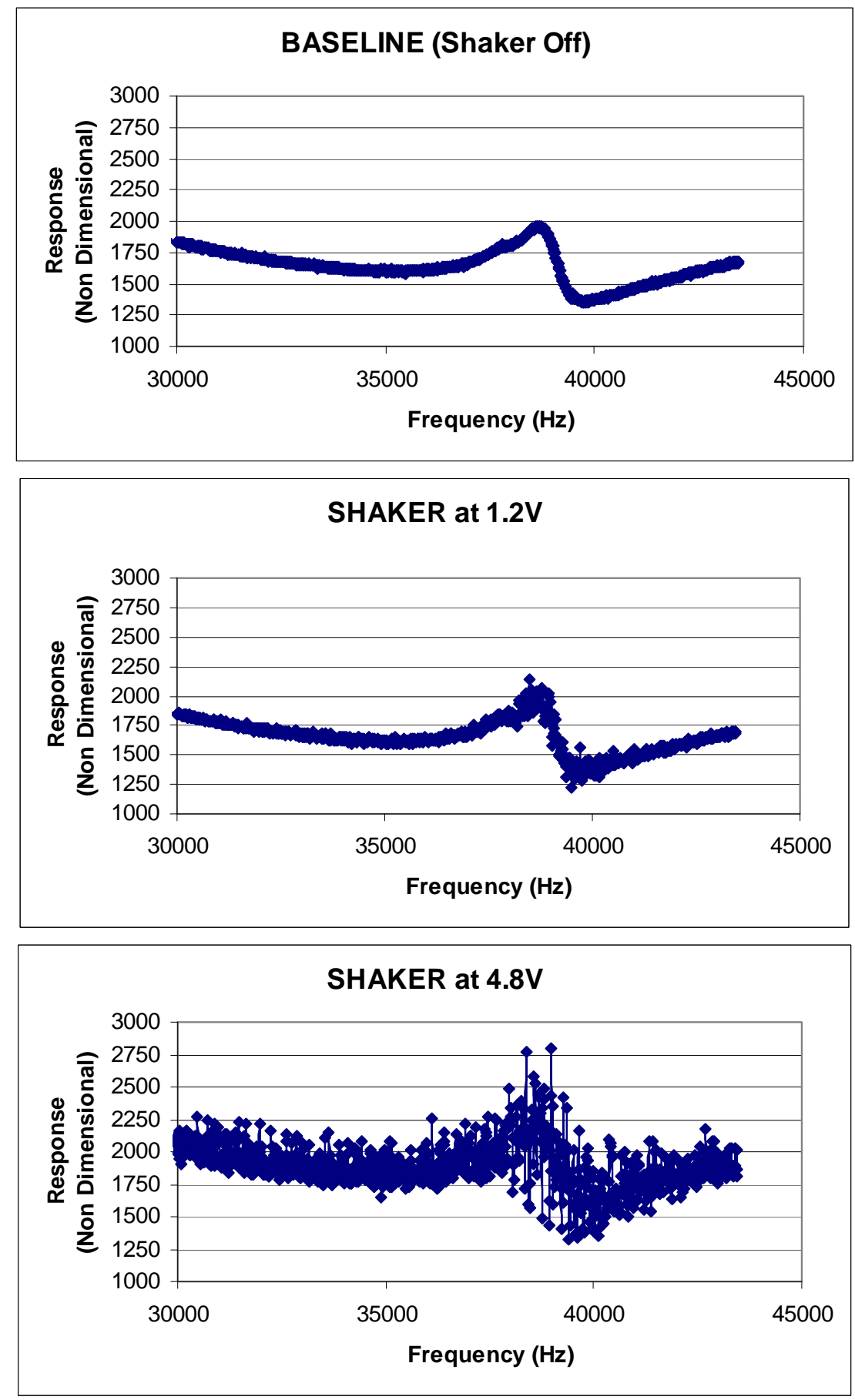

Figure 8. The top graph has the SD signal at 40 in-pound torque with the shaker off, the middle graph has the SD signal with the shaker at a middle setting (voltage $=1.2 \mathrm{~V}$ ), and the bottom graph has the signal with the shaker at the maximum setting (voltage $=4.8 \mathrm{~V})$.

Next, experiments were conducted to quantify the acceleration levels in the previous shaker tests. These tests were conducted by using a reference accelerometer and an intermediate mass so that the resulting mass approximated the mass of the SD accelerometer. The reference accelerometer was chosen because of its higher 
resonance frequency; about $60 \mathrm{KHz}$, and relatively flat response over the frequency range used in the previous SD shaker tests. The sensitivity of the reference accelerometer is $2.5 \mathrm{mV} / \mathrm{g}$.

This calibration of the shaker system indicates a maximum acceleration level of about 3.6 g's, with the source set to 4.8 volts, at the $38 \mathrm{kHz}$ resonance frequency of the SD accelerometer. Other levels were $1.64 \mathrm{~g}$ 's, $0.81 \mathrm{~g}$ 's, 0.41 's, $0.21 \mathrm{~g}$ 's, and $0.06 \mathrm{~g}$ 's at corresponding source settings of 2.4 volts, 1.2 Volts, $600 \mathrm{mV}, 300 \mathrm{mV}$, and 90 $\mathrm{mV}$, respectively.

\section{Analysis}

The SD signal has a definite and repeatable shift downward in resonance frequency as the torque levels are reduced. The overall shift in resonance is about $1.4 \mathrm{kHz}$ as the torque is reduced from 40 in-lbs to $25 \mathrm{in}-\mathrm{lbs}$. A shift of an additional $2.8 \mathrm{kHz}$ occurs as the torque is reduced to zero. The quality of the SD signal, even though noise is present, appears to be sufficiently good so as not to indicate false mounting conditions except in the case of the maximum acceleration, 3.6g. This set of tests allows for the Signal to Noise Ratio (SNR) of the SDA system to be determined for various levels of mechanical noise in the SD frequency band.

The SNR was calculated from the SD accelerometer baseline data, with the shaker off, using Equation 1.

$$
S N R=20 \log _{10} \frac{\sum_{F W H M} A(f)-\text { local } \min [A(f)]}{\sum_{F W H M} N(f)}
$$

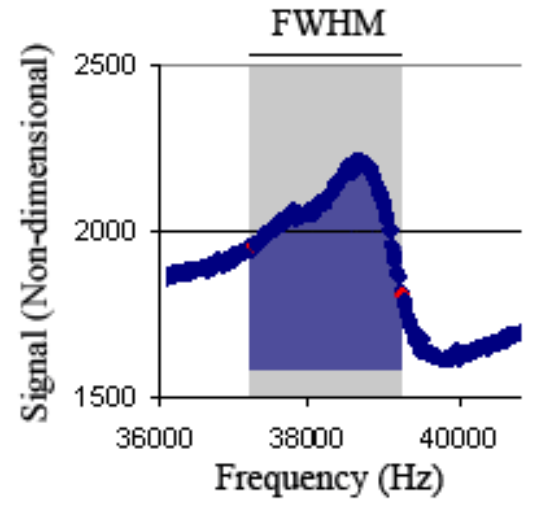

Figure 9. Illustration of the summed signal area, which is in blue.
The signal was obtained by summing over the Full Width Half Maximum, FWHM, of the SD resonant frequency peak. This sum was made using the difference between the SDA frequency response, $A(f)$, and the local minimum of $A(f)$, denoted as local min[A(f)], which removed the bias. An illustration of the signal area that was summed over is shown in blue in Figure 9. The blue area is limited on the top by the signal level and the bottom by the local minimum.

The noise was obtained by taking the absolute value of the difference between the SDA data with the shaker operating at the various levels and the SDA data with the shaker off. This mechanical noise signal, $N(f)$, was then summed over the same frequency band as the signal. The SNR for the 40 in-lbs torque level has been calculated and plotted as a function of acceleration in Figure 10. 


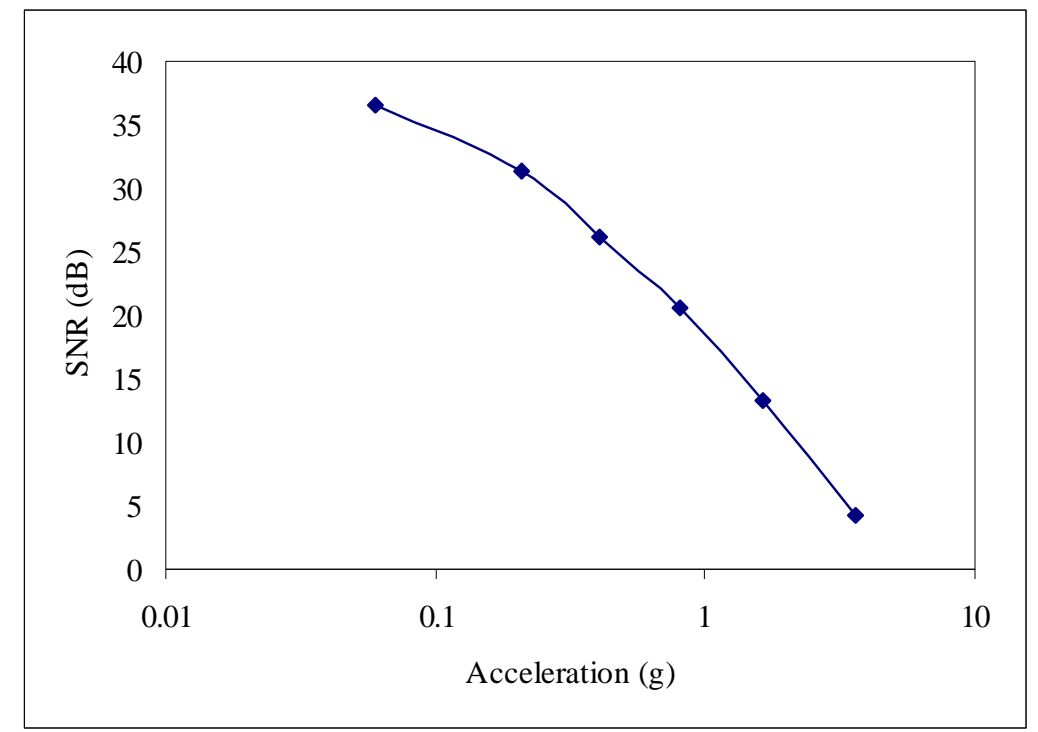

Figure 10. A graph of the SDA SNR as a function of mechanical acceleration.

The graph of the SDA SNR as a function of acceleration shows that the low voltage, $1 \mathrm{Vp}-\mathrm{p}$, SDA system has a reasonable SNR with up to $2 \mathrm{~g}$ 's of acceleration (mechanical noise) in the diagnostic band. Beyond this level of mechanical noise either the signal level must be increased or the frequency peak measurement will be less accurate. If the frequency shifts between a fully torqued accelerometer and a loose accelerometer are far enough apart then lower accuracy may be acceptable. This relation of the SNR to the mechanical noise in the diagnostic band is a key result of this work as it will simplify the evaluation of the feasibility of the SD system, in terms of mechanical noise, for future applications.

\section{Conclusion}

The Self Diagnostic Accelerometer (SDA) system has been shown to be sensitive to multiple accelerometer failure modes and, more importantly, shown to be able to detect a loose accelerometer. In this paper it has been shown that a relatively small and inexpensive SD electronics system can perform the same diagnostic measurements as a expensive and bulky commercial signal analyzer. Also the operation of a low voltage SDA system has been studied in the presence of mechanical noise. The SNR for the system has been measured in terms of acceleration in the diagnostic band and has been shown to be acceptable up to $2 \mathrm{~g}$ 's of acceleration. Future work will include researching the stability of the SD electronics as well as exploring the capability of the system to concurrently compensate for temperature changes while monitoring the condition of the sensor attachment.

\section{Acknowledgments}

The authors wish to recognize the pioneering work of our late colleague, Patrick M. Flanagan, who was instrumental in researching and developing the Self Diagnostic Accelerometer system.

\section{References}

\footnotetext{
${ }^{1}$ Davidson, M. and Stephens, J., “Advanced Health Management System for the Space Shuttle Main Engine”, 40th AIAA/ASME/SAE/ASEE Joint Propulsion Conference, Fort Lauderdale, Florida, July 11-14, 2004.

${ }^{2}$ Simon, D., Garg, S., Hunter, G., Guo, T.-H., and Semega, K.,’'Sensor Needs for Control and Health Management of Intelligent Aircraft Engines”, NASA/TM-2004-213202.

${ }^{3}$ Atherton, W. J. and Flanagan, P. M., " A Self Diagnostic System for Piezoelectric Sensors ", Proceedings of 25th AIAA/ASME/SAE/ASEE Joint Propulsion Conference, Monterey, Ca., July 10-12, 1989

${ }^{4}$ Flanagan, P. M and Atherton, W. J., "Developing a Self-Diagnostic System for Piezoelectric Sensors", Proceedings of 26th AIAA/ASME/SAE/ASEE Joint Propulsion Conference, Orlando, Fl., July 16-18, 1990

${ }^{5}$ Flanagan, P. M, "In-Situ Measurement of Sensitivity for a Piezoelectric Sensor", Proceedings of 27th AIAA/ASME/SAE/ASEE Joint Propulsion Conference, Sacramento, Ca., June 24-26, 1991
} 
${ }^{6}$ Flanagan, P. M, "Design of a Self-Diagnostic Beam-Mode Piezoelectric Accelerometer", Proceedings of 28th AIAA/ASME/SAE/ASEE Joint Propulsion Conference, Nashville, Tenn., July 6-8, 1992

${ }^{7}$ Flanagan, P. M, "Dedicated System for a Self-Diagnostic Piezoelectric Accelerometer", Proceedings of 30th AIAA/ASME/SAE/ASEE Joint Propulsion Conference, Indianapolis, In., June 27-29, 1994

${ }^{8}$ Flanagan, P. M, "Design of a Self-Compensation System for Piezoelectric Accelerometers", Proceedings of 32nd AIAA/ASME/SAE/ASEE Joint Propulsion Conference, Lake Buena Vista, Fl., July 1-3, 1996

${ }^{9}$ Flanagan, P. M., Lekki, J., 'A Self-Diagnostic System for the M6 Accelerometer', Proceedings of 37th AIAA/ASME/SAE/ASEE Joint Propulsion Conference, Sault Lake City, Utah 2001.

${ }^{10}$ Jiang, Q., Cross, L., "Effects of porosity on electric fatigue behaviour in PLZT and PZT ferroelectric ceramics", J. of Mat. Sci., Vol. 28, 1993, pp. 4536-4543.

${ }^{11}$ Kim, S., Jiang, Q., “Microcracking and electric fatigue of polycrystalline ferroelectric ceramics”, Smart Mater. Struct., Vol. 5, 1996, pp. 321-326.

${ }^{12}$ Gao, H., Zhang, T., Tong, P., "Local and global energy release rates for an electrically yielded crack in a piezoelectric ceramic”, J. Mech. Phys. Solids, Vol. 45, No. 4, 1997, pp. 491-510.

${ }^{13}$ Shen, S., Nishioka, T., "Fracture of piezoelectric materials: energy density criterion", Theorectical and Applied Fracture Mechanics, Vol. 33, 2000, pp. 57-65.

${ }^{14}$ Wang, H., Singh, R., “Crack propagation in piezoelectric ceramics: Effects of applied electric fields”, J. Appl. Phys., Vol. 81, No. 11, 1997, pp. 7471-7479. 